\title{
RECENT RESULTS ON DIFFRACTION AT HERA*
}

\author{
Grzegorz P. GaCH \\ on behalf of the H1 and ZEUS collaborations \\ Faculty of Physics and Applied Computer Science \\ AGH University of Science and Technology \\ 30 Mickiewicza Av., 30-059 Kraków, Poland
}

(Received May 26, 2014)

\begin{abstract}
Data in which a proton is tagged along the beampipe after the primary interaction represents the cleanest way to measure diffraction. Data from both $\mathrm{H} 1$ and ZEUS collaborations on inclusive diffractive deep inelastic scattering, with a tagged proton, have been combined. Inclusive diffractive deep inelastic scattering can be considered as the exchange of a colourless object and is amenable to QCD fits in which the structure of this object, sometimes called a Pomeron, is determined. Given such processes are expected to factorise, such a Pomeron structure can be used to predict the rates of jet production in e.g. diffractive photoproduction. The comparison of jet data with the predictions of next-to-leading order QCD are discussed in the context of factorising breaking. Another way to study diffraction is through the production of vector mesons which is also characterised by a colourless exchange with the proton which in lowest order QCD is realised as a colourless gluon pair. Therefore, consideration of the dependence of the $J / \psi$ cross section on the photon-proton centre-of-mass energy is related to the rise of the square of the gluon density towards low values of Bjorken $x$. Comparisons of HERA data with such theories and other data are presented.
\end{abstract}

DOI:10.5506/APhysPolBSupp.7.519

PACS numbers: 13.60.-r

\section{Introduction}

Neutral current lepton-proton interactions have been studied from the beginning of HERA accelerator operation. The process can be described in terms of exchanged boson-proton centre-of-mass energy, $W$, the Bjorken

* Presented at "Excited QCD 2014", Bjelašnica Mountain, Sarajevo, Bosnia and Herzegovina, February 2-8, 2014. 
scaling variable, $x$, squared four-momentum transfer at the proton vertex, $t$, fraction of the incoming lepton energy taking part in the interaction, $y$, and negative squared four-momentum transfer at the lepton vertex called virtuality, $Q^{2}$.

Diffractive neutral current lepton-proton interaction is a process that conserves quantum numbers of incoming particles, i.e. lepton and proton or its dissociated state with proton quantum numbers emerges from a collision. Such process can occur when an object with vacuum quantum numbers called Pomeron is exchanged. This implies that between the produced system and scattered or dissociated incoming particles a rapidity gap should be present. This gap is strongly suppressed in a non-diffractive interaction making it another signature of a diffractive process, next to the presence of scattered proton. In order to describe such interaction, two additional kinematic variables are introduced. The first one is a fraction of proton momentum carried by the Pomeron, $x_{\mathbb{P}}$, and the second one is a quantity equivalent to Bjorken $x$ but relative to the Pomeron momentum, $\beta$.

Recent results on three types of diffractive interactions are presented in the following sections: diffractive vector-meson production, inclusive diffraction and diffractive dijet production.

\section{Diffractive vector-meson production}

Photoproduction cross section of $J / \psi$ meson was measured by the H1 Collaboration [1]. The cross section as a function of squared fourmomentum transfer at the proton vertex was determined in elastic production, i.e. when high energy forward proton emerges from the interaction, and in events with proton dissociation. The results are presented in Fig. 1. The elastic cross section decreases much faster with the rise of $|t|$ than in the case of proton dissociation. A simultaneous fit to both processes was performed. Elastic part was parametrised with $d \sigma_{\mathrm{el}} / d t=N_{\mathrm{el}} \exp \left(-b_{\mathrm{el}}|t|\right)$, while dissociative with $d \sigma_{\mathrm{pd}} / d t=N_{\mathrm{pd}}\left(1+b_{\mathrm{pd}}|t| / n\right)^{-n}$. The following $t$-slopes were obtained: $b_{\mathrm{el}}=(4.88 \pm 0.15) \mathrm{GeV}^{-2}, b_{\mathrm{pd}}=(1.79 \pm 0.12) \mathrm{GeV}^{-2}$.

Another vector meson that was recently measured at HERA is $\Upsilon(1 \mathrm{~S})$. The ZEUS Collaboration determined the $t$-slope of this particle [2]. Not only is it the first time a value of this parameter is extracted, but also $t$-slope of the heaviest measured vector meson so far. A compilation of the slopes corresponding to different mesons as a function of scale defined as a sum of boson virtuality and squared rest mass of the particle is presented in Fig. 2. The new measurement extends the scale more than twice, up to $Q^{2}+M^{2} \approx 90 \mathrm{GeV}^{2}$, where the elastic slope equals

$$
\left.b_{\mathrm{el}}=4.3_{-1.3}^{+2.0} \text { (stat. }\right)_{-0.6}^{+0.5} \text { (syst.) } \mathrm{GeV}^{-2} \text {. }
$$



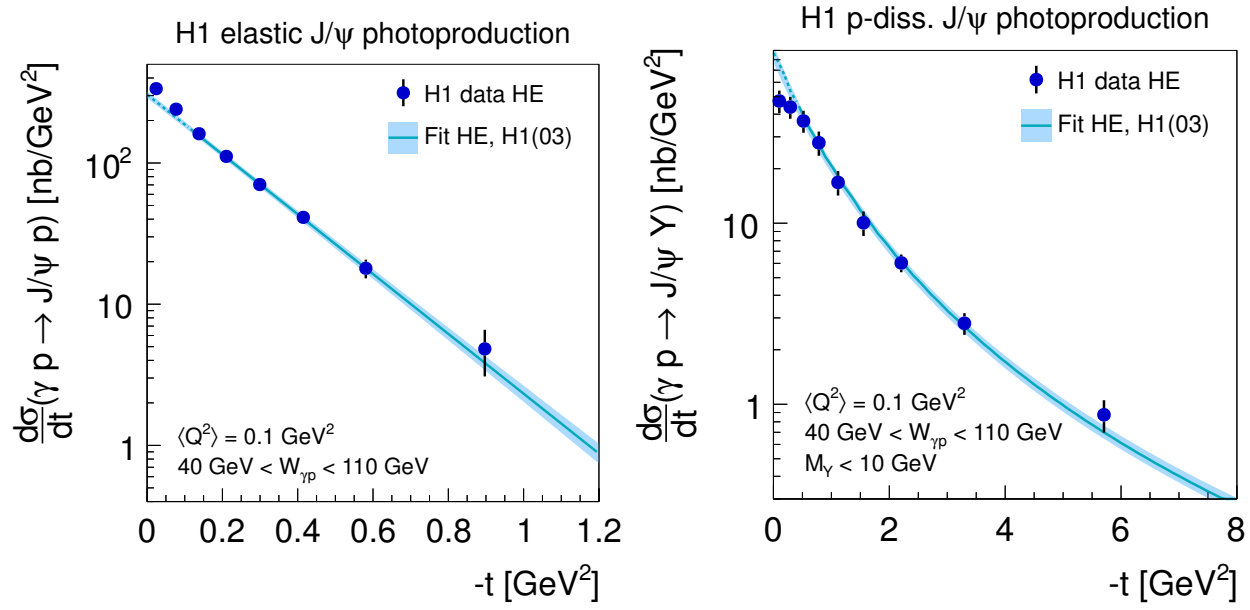

Fig. 1. Measured $t$-slopes of $J / \psi$ elastic (left) and proton dissociative (right) photoproduction together with the fit that was performed simultaneously in the elastic and dissociative regions.
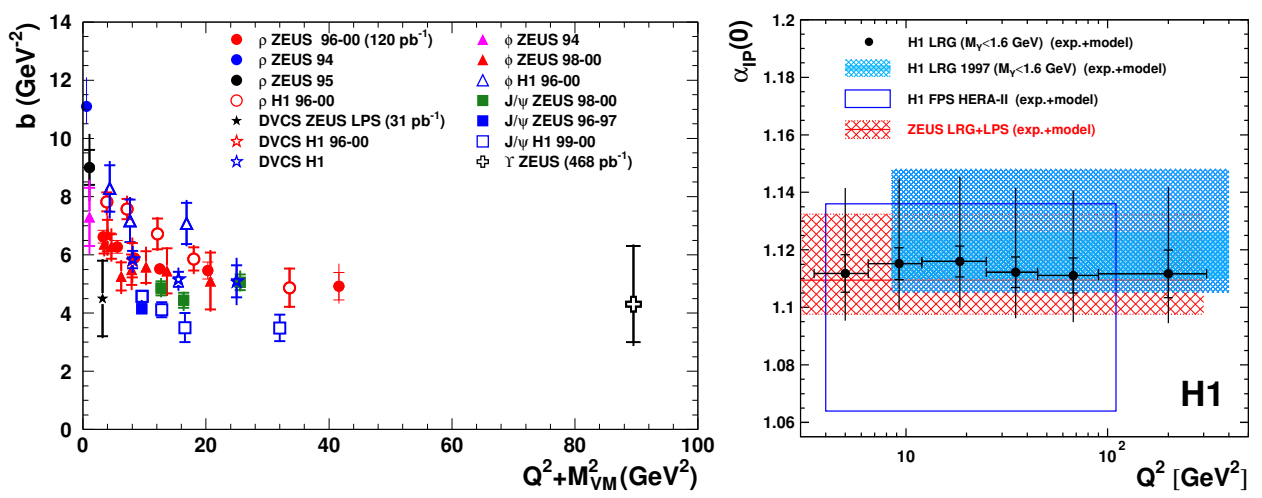

Fig. 2. The left graph presents a set of $t$-slopes in exclusive production of various vector mesons. Recent measurement of $\Upsilon$ 's slope, which was done by ZEUS, extends the measurement region more than twice. The right graph shows Pomeron intercept measured in inclusive diffraction as a function of the exchanged photon virtuality.

\section{Inclusive diffraction}

Differential inclusive lepton-proton diffractive cross section reads

$$
\frac{d^{4} \sigma^{e p \rightarrow e^{\prime} X p^{\prime}}}{d \beta d Q^{2} d x_{\mathbb{P}} d t}=\frac{2 \pi \alpha^{2}}{\beta Q^{4}}\left[1-y+\frac{y^{2}}{2}\right] \sigma_{\mathrm{r}}^{D}\left(\beta, Q^{2}, x_{\mathbb{P}}, t\right)
$$


The reduced fourfold diffractive cross section depends on diffractive structure functions

$$
\sigma_{\mathrm{r}}^{D}\left(\beta, Q^{2}, x_{\mathbb{P}}, t\right)=F_{2}^{D}\left(\beta, Q^{2}, x_{\mathbb{P}}, t\right)-\frac{2 y^{2}}{2-2 y+y^{2}} F_{2}^{L}\left(\beta, Q^{2}, x_{\mathbb{P}}, t\right) .
$$

In deep inelastic scattering, i.e. for $Q^{2}>1 \mathrm{GeV}$, it has been predicted that QCD factorisation holds [1]. It allows to express diffractive cross section of virtual photon-proton interaction with universal diffractive parton densities $\sigma^{D}\left(\gamma^{*} p \rightarrow X p\right) \sim f_{i}^{D}\left(x, Q^{2}, x_{\mathbb{P}}, t\right) \cdot \sigma_{\gamma^{*} i}\left(x, Q^{2}\right)$. Additionally, proton vertex factorisation, which splits parton density functions into Pomeron flux and Pomeron parton densities $f_{i}^{D}\left(x, Q^{2}, x_{\mathbb{P}}, t\right) \sim f_{\mathbb{P} / p}\left(x_{\mathbb{P}}, t\right) f_{i / \mathbb{P}}^{D}\left(\beta, Q^{2}\right)$, is usually assumed.

A reduced diffractive cross section integrated over squared four-momentum transfer at the proton vertex was measured by the H1 Collaboration [3]. The results were compared to the dipole model predictions and calculations based on diffractive parton density functions. The measurement confirms the QCD factorisation in the region of photon virtuality greater than $10 \mathrm{GeV}^{2}$. In the region of small values of $Q^{2}$, perturbative calculations underestimate the cross section. In this region, the dipole model predictions are better. Proton factorisation hypothesis was also tested by the H1 Collaboration. Pomeron intercept, which is Pomeron trajectory value at $t=0$, was measured in inclusive diffractive production in deep inelastic scattering. The results as a function of photon virtuality are presented in Fig. 2. The new results are in agreement with previous ones and no dependence on the virtuality is observed. It supports the proton vertex factorisation hypothesis. The mean value of the intercept was measured to be

$$
\alpha_{\mathbb{P}}(0)=1.113 \pm 0.002(\text { exp. })_{-0.015}^{+0.029}(\text { model }) .
$$

One of the most important diffractive results obtained at HERA is inclusive diffractive cross section [4] combined from H1 [5, 6] and ZEUS [7, 8] measurements. A reduced diffractive cross section $\sigma_{\mathrm{r}}^{D(3)}\left(\beta, Q^{2}, x_{\mathbb{P}}\right)$ was determined as a function of photon virtuality and fraction of the proton momentum carried by the Pomeron in bins of $\beta-x_{\mathbb{P}}$ and $\beta-Q^{2}$ respectively. In Fig. 3 a comparison between separate measurements and the combined one is presented as a function of $Q^{2}$ in three $\beta$ bins. The combined data do provide the most precise determination of the absolute normalisation of the $e p \rightarrow e X p$ cross section. 


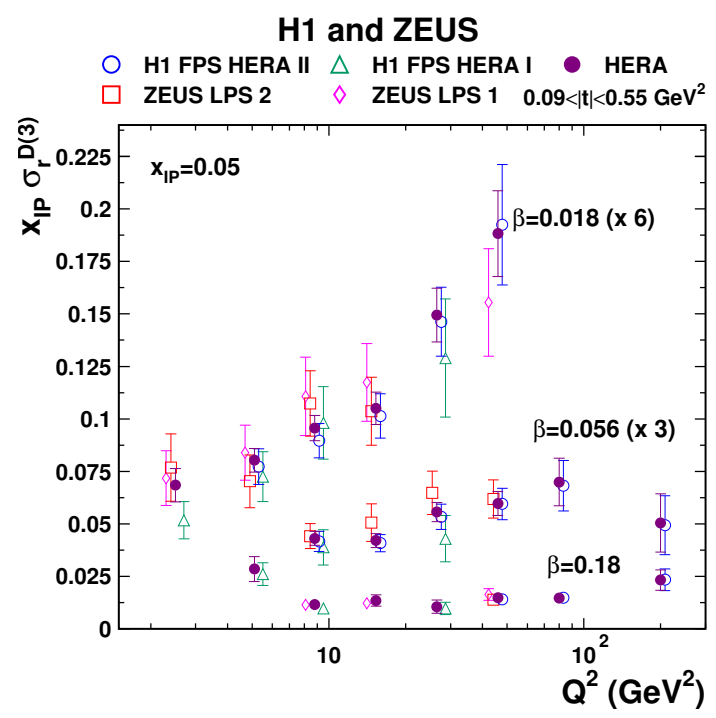

Fig. 3. Inclusive diffractive differential cross section as a function of $Q^{2}$ measured at HERA for $x_{\mathbb{P}}=0.05$ and three values of $\beta$ : 0.018 (cross section scaled by 6 ), 0.056 (scaled by 3), 0.18. The cross section is the result of $\mathrm{H} 1$ and ZEUS measurements combination that leads to unprecedented precision. The full dots labelled HERA show the combined cross section, while all empty symbols show results of single measurements used in the combination.

\section{Diffractive dijet production}

Diffractive dijet production can be calculated in terms of perturbative QCD with the use of diffractive parton density functions. The H1 Collaboration performed extensive studies [9] which support universality of diffractive parton density functions. The measured total cross section is consistent with NLO calculations. In Fig. 4, a comparison between measured and theoretical jet transverse momentum in deep inelastic scattering is presented. The agreement between the two distributions is very good.

Contrary to diffractive dijet production in deep inelastic scattering, NLO calculations of dijet photoproduction $\left(Q^{2}<1 \mathrm{GeV}^{2}\right)$ do not describe the data very well. In $2010, \mathrm{H} 1$ presented measurements based on the rapidity gap selection of diffractive events [10] with cross sections smaller than theoretically predicted. The measurement is now being repeated but with diffractive events selection based on scattered proton tagging. The conclusions from preliminary results are the same as from the previous measurement. The difference in differential cross section between the data and NLO calculations is presented in Fig. 4. 

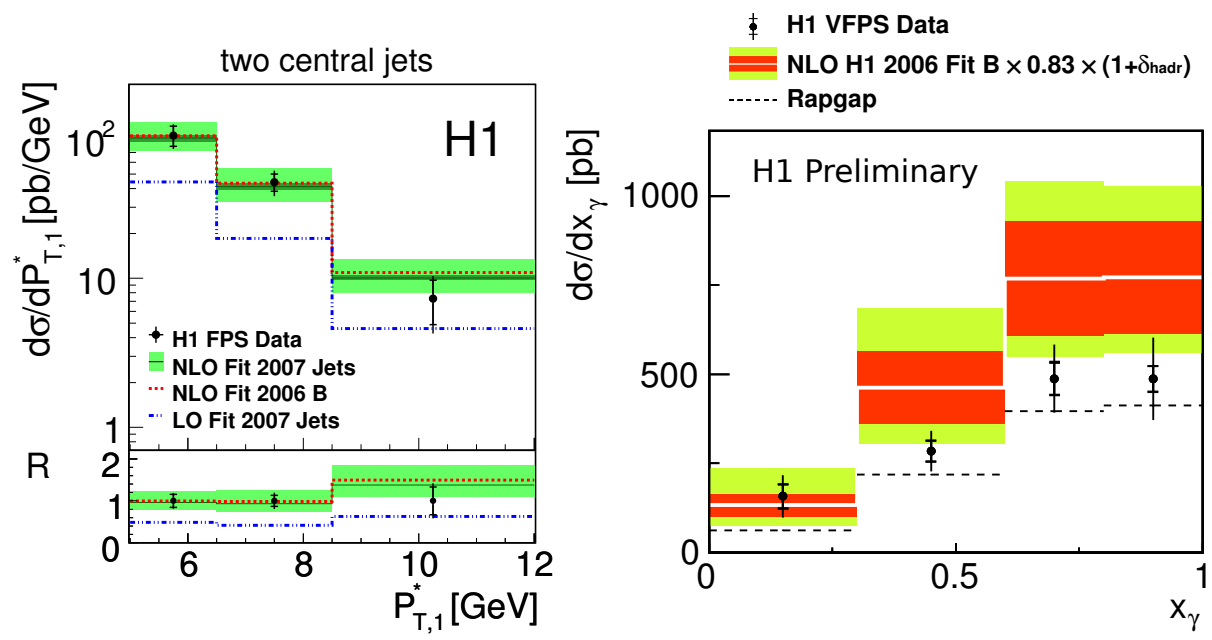

Fig. 4. The left graph shows differential cross section of diffractive dijet production in DIS as a function of the leading jet transverse momentum. $R$ denotes the ratio of the measured cross sections and QCD predictions to the nominal values of the measured cross sections. The right plot presents differential cross section of diffractive dijet photoproduction as a function of longitudinal momentum fraction of the virtual photon taken by the scattered parton. The NLO theoretical prediction is scaled by 0.83 .

This work was partly supported by the Polish National Science Centre under contract No. DEC-2012/06/M/ST2/00428.

\section{REFERENCES}

[1] C. Alexa et al. [H1 Collaboration], Eur. Phys. J. C73, 2466 (2013).

[2] H. Abramowicz et al. [ZEUS Collaboration], Phys. Lett. B708, 14 (2012).

[3] F. Aaron et al. [H1 Collaboration], Eur. Phys. J. C72, 2074 (2012).

[4] F. Aaron et al. [H1 and ZEUS collaborations], Eur. Phys. J. C72, 2175 (2012).

[5] A. Aktas et al. [H1 Collaboration], Eur. Phys. J. C48, 749 (2006).

[6] F. Aaron et al., Eur. Phys. J. C71, 1578 (2011).

[7] S. Chekanov et al. [ZEUS Collaboration], Nucl. Phys. B816, 1 (2009).

[8] S. Chekanov et al. [ZEUS Collaboration], Eur. Phys. J. C38, 43 (2004).

[9] F. Aaron et al. [H1 Collaboration], Eur. Phys. J. C72, 1970 (2012).

[10] F. Aaron et al. [H1 Collaboration], Eur. Phys. J. C70, 15 (2010). 